SHORT REPORT

\title{
Effects on health of prolonged exposure to low concentrations of carbon monoxide
}

\section{L Townsend, R L Maynard}

$\mathrm{T}$ he effects on health of prolonged but low level exposure to carbon monoxide (CO) are unclear. Studies of carbon monoxide exposure focus mainly on short term effects in experimental settings, or on long term effects in cases of accidental poisoning. Exposures in long term case studies are often of unknown levels and duration. Patients are sometimes exposed to short periods of acute intoxication, in addition to the low level, chronic exposure; thus the difficulty in determining which type of exposure is responsible for any subsequent health problems. Anecdotal evidence suggests that chronic exposure to $\mathrm{CO}$ may produce mild neurological effects. Although there are as yet no conclusive studies showing such a correlation, the evidence in its favour is accumulating.

The effects of severe exposure to carbon monoxide are well understood. The very effective competition of carbon monoxide with oxygen for binding sites on haemoglobin with the subsequent left shift of the dissociation curve leads to a reduction in both oxygen transport and release. This produces a range of effects on health. In 1999, a WHO expert group reviewed the subject and reported their conclusions in Environmental Health Criteria 213, Carbon Monoxide. ${ }^{1}$ A short account has been provided by the Expert Panel on Air Quality Standards. ${ }^{2}$ Severe poisoning, leading to a period of unconsciousness, may lead to neurological damage that may be long lasting, though eventual recovery seems to be the general rule. The mechanisms underlying the neurological damage are imperfectly known, but are thought to include an inflammatory response that may be initiated during the reperfusion or recovery phase when blood with increasing levels of oxygen flows into tissues that had hitherto been severely hypoxic. Reperfusion injury is known to play a part in the damage produced by myocardial infarction. ${ }^{3}$ Free radical release may lead to lipoperoxidation, with the release of inflammatory mediators and the subsequent influx of neutrophils along a chemotactic gradient. Damage may be widespread, though the corpus striatum seems particularly vulnerable. The reasons for this are incompletely understood, though the fact that parts of the striatum lie at the boundary of the arterial supply from the anterior and middle cerebral arteries may be significant. The striatum is also reported to have a high oxygen consumption. ${ }^{4}$ Damage to the striatum provides an explanation for the Parkinsonian signs that may follow severe poisoning with carbon monoxide. Other sequelae may include intellectual deterioration, memory impairment, and changes in emotional stability. It has been generally assumed that such effects only occur after severe poisoning.

Short term exposure to high concentrations of carbon monoxide certainly produces changes in intellectual functioning. John Scott Haldane and his son JBS Haldane reported decrements in their ability to do arithmetic calculations as their carboxyhaemoglobin $(\mathrm{COHb})$ levels rose during experimental exposure to $\mathrm{CO}$. Complete recovery of mental functioning post exposure was also noted. ${ }^{5}$ This experience and that of many other experimentalists, has led to the conclusion that transient exposure to $\mathrm{CO}$ does not have long lasting effects on the brain. Of course, most experimental exposures to $\mathrm{CO}$ have tended to be of short duration: up to several hours.

More recently it has been suggested that prolonged exposure (days-months) to low concentrations of CO may have subtle effects on the brain. If true, this is clearly worrying as such exposure may well occur as a result of malfunctioning heating devices in people's homes. Daily exposure, leading to symptoms including headache and malaise are often reported with periods of recovery to normality occurring when exposure stops. Thus recovery during the working day with a recurrence of symptoms during the evening, or recovery during a holiday with deterioration on return, has been reported. ${ }^{6}$ The general expectation of full recovery on removal of the source of carbon monoxide has recently been questioned by Penney and colleagues. ${ }^{6}$ Anecdotal reports of prolonged effects on intellectual functioning are commonthough, again, the general perception seems to be that eventual full recovery is the rule.

\section{IS THERE A PROBLEM IN THE UK? HOW LARGE IS IT?}

The Institute for Environment and Health commented in their 1998 publication on carbon monoxide that "it is likely that many more subacute CO intoxications occur than are brought to the attention of medical practitioners." ${ }^{\prime 7}$ Because of the difficulty in recognising the effects of exposure to low concentrations of CO, there is currently limited knowledge of the size of the problem. In order to assess whether low level carbon monoxide exposure is, in fact, a problem in the UK, two important factors need to be taken into account: the number of people potentially affected by low levels of $\mathrm{CO}$ in their homes, and the severity and likelihood of long term effects.

The World Health Organisation issued the following guidelines for levels of $\mathrm{CO}$ in the air, to prevent blood $\mathrm{COHb}$ levels from rising above $2.5 \%$.

- $100 \mathrm{mg} / \mathrm{m}^{3}$ (87.1 ppm) for 15 minutes

- $60 \mathrm{mg} / \mathrm{m}^{3}$ (52.3 ppm) for 30 minutes

- $30 \mathrm{mg} / \mathrm{m}^{3}$ (26.1 ppm) for 1 hour

- $10 \mathrm{mg} / \mathrm{m}^{3}$ (8.7 ppm) for 8 hours.

The Expert Panel on Air Quality Standards reported in 1994 that blood $\mathrm{COHb}$ levels of $2.5-4 \%$ were associated with "decreased short-term maximal exercise duration in young healthy men."' ${ }^{2}$ Levels of 2.7-5.1\% COHb were associated with "decreased exercise duration due to increased chest pain (angina) in patients with ischemic heart disease". Levels of $2-20 \% \mathrm{COHb}$ led to "equivocal effects on visual perception, audition, motor and sensorimotor performance, vigilance, and other measures of neurobehavioural performance".

Smokers are an interesting exception to this. While non-smokers generally have COHb levels of about $0.5 \%$, as a result of normal endogenous production of carbon monoxide, smokers can have COHb levels of up to about $13 \%$, yet they seem not to experience the types of effects that do those exposed to ambient $\mathrm{CO}$ who attain similar $\mathrm{COHb}$ levels. Some 
have used this observation to argue that low levels of carbon monoxide do not have neurological effects. However, it cannot be disputed that COHb levels of well below 13\% are correlated with decreased mental performance (this will be discussed later). Without understanding why smokers are seemingly "immune" to such levels of COHb, it is clear that they are an exception to the general rule and should not be used to estimate the effects of low level carbon monoxide exposure on non-smokers in the home.

As exposure above $9 \mathrm{ppm}$ for longer than eight hours leads to $\mathrm{COHb}$ levels of above $2.5 \%$, health effects might occur at such levels. In the case of a malfunctioning gas heater, exposure would potentially occur for as long as the problem remained undetected, lasting weeks or even months. In a study by Amitai et al, the authors reported the results of tests carried out by the US National Bureau of Standards. ${ }^{8}$ They found that properly functioning kerosene heaters in poorly ventilated rooms could produce CO levels of 46-98 ppm.

Several studies have attempted to determine levels of carbon monoxide in people's homes. ${ }^{910}$ Schaplowsky et al measured blood COHb levels in about 3000 children in the USA, as well as CO concentrations in 1820 randomly selected homes. ${ }^{9}$ They found that $22.5 \%$ of children $(n=517)$ had blood $\mathrm{COHb}$ levels above $3 \%$. Highest levels were around $8 \%$. Of the homes tested for $\mathrm{CO}$ concentrations, about $17 \%$ $(\mathrm{n}=306)$ had levels above $10 \mathrm{ppm}$. These raised levels of CO were primarily caused by defective or improperly vented fuel burning appliances. However, passive smoking was not taken into account, and it is known that this can play an important part in raising indoor concentrations of CO. Nevertheless, this study suggests that a large proportion of the population may be unknowingly exposed to carbon monoxide levels above those considered safe. While this study was conducted in the United States, Dr David Penney points out on his website that there is potentially more of a problem in the UK than in the USA. ${ }^{11}$ In the UK the use of gas appliances is more common, and homes tend to be older and therefore furnished with older appliances.

Dolan et al examined blood COHb levels in patients with flulike symptoms at a hospital in Kentucky. ${ }^{10}$ In 13 of 55 patients $(23.6 \%)$ who were tested, COHb levels above $10 \%$ were reported. As the patients who participated in the study were self selected, they may not represent a characteristic proportion of all patients who experience flu-like symptoms. However, it is possible that a proportion of patients reporting to their GPs with such symptoms are in fact suffering from the effects of low concentrations of $\mathrm{CO}$ in their home environment.

As it seems likely that many people are exposed to CO in their homes, it is important to determine what effects this may have on their health. For normal, healthy individuals, the effects may be small or non-existent. However certain individuals, such as children, the elderly, pregnant women, and people with chronic diseases involving impaired blood supply to the heart and brain may be more susceptible to increased levels of CO.

\section{ARE OTHER MECHANISMS INVOLVED?}

The mechanism of carbon monoxide toxicity is primarily through hypoxia. When inhaled, $\mathrm{CO}$ is absorbed through the lungs, where it binds with haemoglobin to form COHb. As its affinity for $\mathrm{Hb}$ is 234 times greater than that of oxygen, the amount of oxygen in the blood becomes greatly reduced, causing a hypoxic state. Furthermore, once CO is bound, it alters the dissociation curve of oxyhaemoglobin, thus reducing the rate at which oxygen is released to the cells. Tissues with a greater requirement for oxygen, such as the brain and heart, are more severely affected by hypoxia. ${ }^{12}$

While hypoxia can account for some of the effects of $\mathrm{CO}$ exposure, it is not consistent with certain observations. In many cases, symptoms persist or even appear after $\mathrm{COHb}$ lev- els have returned to normal. These delayed neuropsychological sequelae can appear 2-40 days after exposure. They include symptoms seen during exposure, such as lethargy, headaches, and concentration problems, as well as other symptoms, such as amnestic syndromes, dementia, psychosis, and parkinsonism. These cannot be explained by hypoxia alone, as one would expect an improvement of symptoms once the hypoxic stress is removed. ${ }^{13}$ Another indication that a toxic mechanism besides hypoxia might be involved is that there is no clear correlation between COHb levels and the effects on health. ${ }^{7}$ Levels of $\mathrm{COHb}$ do not always correspond to the intensity of symptoms and cannot be used to reliably predict the patient's outcome. Feldman also noted that the morphological changes seen in brain tissue at autopsy could not be caused by CO alone. ${ }^{4}$

So far, no direct link between the role of $\mathrm{CO}$ as a messenger and the long term effects reported by some patients has been found. However, the discovery that CO could be involved in biological pathways within cells provides the potential for a mechanism. Haem oxygenase-1 catalyses the breakdown of haem containing proteins with the subsequent release of CO. Haem oxygenase- 1 is produced in the brain in response to hypoxia, and thus endogenously produced $\mathrm{CO}$ could play a part in the response to hypoxia itself induced by exogenous $\mathrm{CO}^{4}$

Haem oxygenase- 1 is found in several brain regions, including the hippocampus and the olfactory neurones, indicating the possible role of $\mathrm{CO}$ in these regions. ${ }^{14}{ }^{15}$ It has been suggested that $\mathrm{CO}$ might have a role in long term potentiation, a process necessary for the formation of memories. ${ }^{14}$ It is of interest that the symptoms reported after exposure to $\mathrm{CO}$ have included memory deterioration and ocular effects. ${ }^{6}$

Thom and Ischiropoulos studied human endothelial cells incubated with environmentally relevant levels of CO (10-20 ppm). ${ }^{16}$ They found that the cells released nitric oxide, causing oxidative stress capable of affecting cell physiology.

While the effects of carbon monoxide on cellular function are not fully understood, there is enough evidence to indicate that hypoxia, per se, might not be the only process involved in CO pathology.

\section{OTHER EFFECTS OF LOW LEVELS: HEART DISEASE, LOW BIRTH WEIGHT}

The difficulty in determining whether very low levels of CO produce long term health effects arises partly from the fact that these levels generally do not produce consistent symptoms. People with blood COHb levels of around 5\% or below generally experience no symptoms; this is true in cigarette smokers in whom $\mathrm{COHb}$ concentrations may reach $12 \%$. However, there is substantial evidence that such low levels have other health effects. Epidemiological studies have shown that levels of $\mathrm{CO}$ in the air are correlated with mortality rates and hospital admissions for heart failure. This has important public health implications, as heart disease is a leading cause of death in the USA and the UK.

In 1995, Morris et al studied the role of ambient air pollution in connection with heart failure in the elderly. ${ }^{17}$ They found that an increase in ambient carbon monoxide levels led to a corresponding increase in admissions to hospital with heart failure. They suggest that ambient $\mathrm{CO}$ could precipitate or exacerbate already existing health conditions. A similar study by Burnett et al supported this hypothesis. ${ }^{18}$ In this study of the 10 largest Canadian cities, the authors found a positive association between ambient levels of $\mathrm{CO}$ and hospital admission rates for congestive heart failure, specifically in the elderly. The authors reported a stronger correlation with CO than with other pollutants, and mentioned that this observation was in agreement with two other studies. However, as with any study of this kind, the authors were unable to rule out the possibility that other factors were influencing their results, as it was difficult to dissociate $\mathrm{CO}$ from other pollutants. Nonetheless, 
after adjustment for confounding factors, an association between ambient $\mathrm{CO}$ levels and hospital admissions remained.

Experimental studies have shown that low levels of $\mathrm{CO}$ adversely affect patients with heart disease when exercising. Allred et al examined the effect of $\mathrm{CO}$ on myocardial ischaemia. ${ }^{19}$ They found that during exercise, COHb levels of $2 \%$ or $4 \%$ caused a decrease in the length of time (by $4.2 \%$ and $7.1 \%$ respectively) before the onset of angina.

Thom and Ischiropoulos have suggested a possible mechanistic connection between $\mathrm{CO}$ and atherosclerosis, a disease causing deposits of fat, cholesterol, cells, and connective tissue in blood vessels. ${ }^{16}$ By exposing blood platelets to varying concentrations of $\mathrm{CO}$, the authors showed that relatively low levels caused the cells to release nitric oxide. At concentrations of 50 or $100 \mathrm{ppm}$, peroxynitrite was produced. Although this may not be relevant at ambient levels of $\mathrm{CO}$, it is interesting that oxidative stress (such as that caused by peroxynitrite) is known to damage the vascular endothelium. The study therefore shows another possible biochemical mechanism for $\mathrm{CO}$ mediated pathology.

Morris has questioned the apparent discrepancy between the relatively high levels of $\mathrm{CO}$ needed to produce results in experimental studies, and the effects of small changes in ambient levels of $\mathrm{CO}$ on hospital admissions ${ }^{20}$ Morris suggests that this may reflect the difference between ambient levels and personal exposure, which can be much higher near roads or in traffic than would be predicted from "background" levels. Furthermore, the persons affected the most by increases in ambient carbon monoxide are those already prone to heart disease. A rise in CO may exacerbate their symptoms, leading to hospitalisation. Experimental studies generally involve healthy individuals. Interaction between CO and other stressors such as raised ambient temperature should also be considered. Morris reported that above $75^{\circ} \mathrm{F}\left(24^{\circ} \mathrm{C}\right)$, ambient $\mathrm{CO}$ had little effect on congestive heart failure hospital admissions. Thus, lower temperatures exacerbate the effects of CO on hospital admissions. Keatinge et al have shown that reductions in ambient temperature cause changes in clotting factors in blood and had linked this with the increase in myocardial infarctions and strokes seen in cold weather. ${ }^{21}$ It is tempting to speculate that carbon monoxide could be playing an additive or, possibly, synergistic role. The interaction between CO and other factors in the environment may help explain why laboratory and chamber studies often report only small effects.

A correlation between maternal exposure to $\mathrm{CO}$ and low birth weight has also been reported..$^{22}$ Exposure to higher levels of CO during the last trimester of pregnancy was associated with lower birth weight in babies (odds ratio $=1.22$ ). The effect on birth weight of increased ambient $\mathrm{CO}$ was as large as the effect of the mother smoking a pack of cigarettes per day during pregnancy. While maternal smoking habits were not accounted for in this study, the authors adjusted their results for other confounding factors (maternal age, race, and education) which are themselves correlated with smoking behaviour.

The studies discussed above address different issues from long term neurological effects. However, there are important similarities. For example, the effects of ambient $\mathrm{CO}$ indicate that even low levels of CO can have important health effects. It is also true that in none of the effects listed above, nor in the suggested neurological effects, is the mechanism of effects understood. Though interference with oxygen availability at the cellular level may underlie some of the effects, the possible involvement of carbon monoxide as a transmitter substance cannot be ruled out. Finally, the potential public health issues arising from $\mathrm{CO}$ as an ambient air pollutant or from low levels of $\mathrm{CO}$ in the home may be similar.

EXPOSURE TYPES: CASES FOR LONG TERM EFFECTS Short term effects in experimental settings

Carbon monoxide exposure at varying levels leads to a range of different effects, from headaches and lethargy to coma, seizures, and death. ${ }^{8}$ There is no doubt that acute levels of CO lead to severe neurotoxicity with lasting effects. ${ }^{7}$ However, the evidence for lasting effects occurring at much lower levels of exposure is poor. While there are as yet no epidemiological studies on this, there is experimental evidence that $\mathrm{CO}$ can affect the brain even at very low levels. ${ }^{83}$ Case studies of individuals who have experienced neurological and psychological problems post-CO exposure have also been reported. ${ }^{64}$ As early as 1963, Schulte reported the results of psychological testing of volunteers exposed to $\mathrm{CO} .{ }^{25} \mathrm{He}$ reported that certain psychomotor abilities were affected at $\mathrm{COHb}$ levels of $5 \%$. In tests of cognitive abilities, subjects showed an increasing number of errors and completion time as COHb levels increased, the effect becoming apparent at $5 \% \mathrm{COHb}$. These results indicate that even before clinical symptoms are reported (around 20\% COHb), cognitive skills can be affected. Putz (1979) used a dual task test to measure the effect of low level carbon monoxide exposure on subject performance. ${ }^{23}$ The advantage of a dual task test is that it reduces the possibility that subjects will compensate for the $\mathrm{CO}$ effects and negate the test. Providing two information sources increases the difficulty of the test as a whole, without the need for more complex tests which are often more difficult to assess. Subjects were exposed to CO levels which produced $\mathrm{COHb}$ levels of below $1 \%, 3 \%$, or $5 \%$ over a four hour exposure time. The performance of subjects with $5 \% \mathrm{COHb}$ was worse than that of the other two groups, specifically in terms of response times and efficiency.

Many other studies have investigated performance on different tracking tasks, including Benignus et al (1990), who observed no difference in performance between subjects with $5 \%, 12 \%$, or $17 \% \mathrm{COHb}^{26}$ The results of other studies have been inconsistent. Often, effects appear to be small and do not achieve statistical significance. Benignus et al suggest that this may be a result of the wide range of psychological tests used, and the importance of the rate of formation of $\mathrm{COHb}$, which varies depending on experimental design.

Amitai et al assessed the effect of low level carbon monoxide exposure on higher cognitive functions in university students. ${ }^{8}$ They noted that subjects with COHb levels of $1-10 \%$ (ambient CO concentrations varied from 17 to $100 \mathrm{ppm}$ ) had significantly lower scores on many parts of the test than the study group. While their results suggest that low levels of $\mathrm{COHb}$ are correlated with poor performance on certain tests involving higher cognitive functions, they again do not provide conclusive data. This study, for example, was criticised for omitting to administer an intelligence test before the exposure, for using ambient CO measurements as a marker for $\mathrm{COHb}$, instead of measuring $\mathrm{COHb}$ directly, and for misinterpreting the test results. ${ }^{27}$

From the variety of different results obtained, it seems clear that further, more standardised testing is necessary. In many cases $\mathrm{CO}$ appears to affect the test taking ability of individuals exposed during the tests. However, these results have been contested, and other tests have shown no difference between control and study groups. Ethical questions about such studies may be raised. It is difficult to imagine that ethical approval would be given for a study that sought to test the hypothesis that exposure to carbon monoxide produced long lasting neurological damage.

\section{Long term effects: case studies}

Ryan (1990) presented the case of a woman who complained of headaches, lethargy, and memory problems over a period of three years. ${ }^{24}$ Levels of CO in her basement were found to be 180 ppm. COHb levels were not recorded. The patient performed very poorly on tests that measured new learning ability and memory for new material, her scores being in the lowest fifth percentile. She also showed poor performance on tests for visual memory processes. The patient reported feelings of depression and anxiety, as well as headaches and dizziness, even after the 
Main messages

- The toxicology of $\mathrm{CO}$ is more complicated than generally thought.

- Accidental CO poisoning remains a problem in the UK.

- Diagnosis of poisoning by low concentrations of CO is difficult.

- Associations between outdoor levels of $\mathrm{CO}$ and exacerbation of heart disease are appearing.

- Long term exposure to low concentrations may cause neurological damage.

furnace had been repaired. This case suggests that the development of long term neurological symptoms can occur even without loss of consciousness.

Myers et al (1998) reported seven cases of chronic, subacute CO poisoning, in which patients experienced many similar symptoms, even after the source of CO was identified and removed. $^{28}$ The neuropsychological symptoms reported included changes in memory, sleep, vision, sense of smell and sense of direction, anxiety, psychomotor dysfunction, and balance problems. The authors point out that despite the many symptoms reported in their study and in others, "it is difficult to understand how chronic exposure to low levels of a toxic substance can produce these marked cognitive and psychological changes without marked neurological effects".

These case studies indicate that lasting neurological effects can occur after prolonged exposure to carbon monoxide, even when the victims are no longer exposed.

\section{CONCLUSION}

Evidence that exposure to low concentrations of carbon monoxide can affect a number of organ systems is accumulating. It is, perhaps, easiest to explain effects on the heart in subjects with incipient myocardial ischaemia. Less easy to explain are effects on the central nervous system; that these effects may not be accurately predicted on the grounds of blood $\mathrm{COHb}$ concentration does, however, seem increasingly clear. Whether long term exposure to low concentrations of carbon monoxide can produce long lasting effects on the brain does not yet seem to be settled. If such effects do occur, the impact on public health may be large: many homes are heated with gas appliances and a significant number by solid fuel; failures are inevitable and known to be common, and thus a significant number of people must be being exposed to levels of carbon monoxide in excess of those found in ambient air. Even if only a modest proportion of those exposed sustained effects, the impact on public health may be significant. These findings may have implications for the setting of occupational exposure limits. The Health and Safety Executive recommend a limit of $30 \mathrm{ppm}$, which can cause $\mathrm{COHb}$ levels to rise above $2.5 \%$ in less than one hour. However, it should be noted that the evidence for low level effects of carbon monoxide does not arise from occupational exposure studies. The patterns of exposure of people exposed in their homes may be quite different from those exposed occupationally.

\section{Authors' affiliations}

C L Townsend, R L Maynard, Department of Health, London, UK

The views expressed here are those of the authors and should not be taken as those of the UK Department of Health

Correspondence to: Prof. R L Maynard, Department of Health, Room $661 \mathrm{C}$, Skipton House, 80 London Road, London SE 1 6LH, UK; robert.maynard@doh.gsi.gov.uk

Accepted 10 April 2002

\section{Policy implications}

- Increased public awareness of the dangers of $\mathrm{CO}$ is needed.

- Maintenance of appliances that can malfunction and generate $\mathrm{CO}$ is vital, especially if prolonged exposure to low concentrations is to be avoided.

- More research on the effects of $\mathrm{CO}$ as an outdoor air pollutant is needed.

\section{REFERENCES}

1 WHO. Environmental Health Criteria 213: Carbon Monoxide, 2nd edn. Finland: World Health Organisation, 1999

2 Department of the Environment Expert Panel on Air Quality Standards. Carbon monoxide. London: HMSO, 1994.

3 Poole Wilson PA, Snyder PH. Cardiovascular disease: physiological considerations: biochemistry and cellular physiology of heart muscle. Oxford textbook of medicine, 3rd edn. Oxford: Oxford University Press, 1996:2143-52.

4 Feldman RG. Occupational and environmental neurotoxicology. Philadelphia, PA: Lippencott-Raven, 1999:378-99.

5 Maynard RL, Waller R. Carbon monoxide. In: Holgate ST, Samet JM, Koren HS, Maynard RL, eds. Air pollution and health. London: Academic Press, 1999:749-96.

6 Penney DG. Chronic carbon monoxide poisoning. In: Penney DG, ed. Carbon monoxide toxicity. London: CRC Press, 2000:393-418.

7 Institute for Environment and Health. IEH assessment on indoor air quality in the home (2): carbon monoxide. Norwich: Page Bros, 1998.

8 Amitai Y, Zlotogorski Z, Golan-Katzav V, et al. Neuropsychological impairment from acute low-level exposure to carbon monoxide. Arch Neurol 1998;55:845-8

9 Schaplowsky AF, Oglesbay FB, Morrison JH, et al. Carbon monoxide contamination of the living environment: a national survey of home air and children's blood. J Environ Health 1974;36:569-73.

10 Dolan MC, Haltom TL, Barrows GH, et al. Carboxyhemoglobin levels in patients with flu-like symptoms. Ann Emerg Med 1987;16:782-6.

11 Penney DG. http://www. phymac.med.wayne.edu/FacultyProfile/ penney/COHQ/COl.htm

12 Piantadosi CA. Toxicity of carbon monoxide: hemoglobin vs. histotoxic mechanisms. In: Penny DG, ed. Carbon monoxide. London: CRC Press, 1997: 163-86

13 Thom SR, Taber RL, Mendiguren II, et al. Delayed neuropsychologic sequelae after carbon monoxide poisoning: prevention by treatment with hyperbaric oxygen. Ann Emerg Med 1995;25:474-80.

14 Barinaga M. Carbon monoxide: killer to brain messenger in one step [letter]. Science 1993;259:309.

15 Verma A, Hirsh DJ, Glatt CE, et al. Carbon monoxide: a putative neura messenger. Science 1993;259:381-3.

16 Thom SR, Ischiropoulos H. Mechanism of oxidative stress from low levels of carbon monoxide. Health Effects Institute Research Report Number 80. 1997.

17 Morris RD, Naumova EN, Munasinghe RL. Ambient air pollution for congestive heart failure among elderly people in seven large US cities. Am J Public Health 1995;85:1361-5.

18 Burnett RT, Dales RE, Brook JR, et al. Association between ambient carbon monoxide levels and hospitalizations for congestive heart failure in the elderly in 10 Canadian cities. Epidemiology 1997;8:162-7.

19 Allred EN, Bleecker ER, Chaitman BR, et al. Short-term effects of carbon monoxide exposure on the exercise performance of subjects with coronary artery disease. N Engl J Med 1989;321:1426-32.

20 Morris RD. Low-level carbon monoxide and human health. In: Penney DG, ed. Carbon monoxide toxicity. London: CRC Press, 2000:381-91.

21 Keatinge WR, Coleshaw SRK, Cotter F, et al. Increases in platelet and red blood cell counts, blood viscosity, and arterial pressure during mild surface cooling: factors in winter mortality from coronary and cerebral thrombosis in winter. BM 1989;289:1405-8.

22 Ritz B, Yu F. The effect of ambient carbon monoxide on low birth weight among children born in southern California between 1989 and 1993. Environ Health Perspect 2000;107:17-25.

23 Putz VR. The effects of carbon monoxide on dual-task performance. Human Factors 1979;21:13-24

24 Ryan CM. Memory disturbances following chronic, low-level carbon monoxide exposure. Arch Clin Neuropsychol 1990:5:59-67.

25 Schulte JH. Effects of mild carbon monoxide intoxication. Arch Environ Health 1963;7:524-30.

26 Benignus VA, Muller KE, Smith MV, et al. Compensatory tracking in humans with elevated carboxyhemoglobin. Neurotoxicol Teratol 1990; 12:105-10.

27 Bleeker ML. The mere presence of low levels of carboxyhemoglobin is not causal proof of altered neuropsychological performance [letter]. Arch Neurol 1999;56:1299.

28 Myers RAM, DeFazio A, Kelly MP. Chronic carbon monoxide exposure: a clinical syndrome detected by neuropsychological tests. J Clin Psychol 1998;54:555-67. 